\title{
Turbulent Duct Flow Controlled with Spanwise Wall Oscillations
}

\author{
Steffen Straub $^{1}$ - Ricardo Vinuesa ${ }^{2}$ - Philipp \\ Schlatter $^{2}$. Bettina Frohnapfel ${ }^{1}$. Davide Gatti ${ }^{1}$
}

Received: date / Accepted: date

\begin{abstract}
The spanwise oscillation of channel walls is known to substantially reduce the skin-friction drag in turbulent channel flows. In order to understand the limitations of this flow control approach when applied in ducts, direct numerical simulations of controlled turbulent duct flows with an aspect ratio of $A R=3$ are performed. In contrast to channel flows, the spanwise extension of the duct is limited. Therefore, the spanwise wall oscillation either directly interacts with the duct side walls or its spatial extent is limited to a certain region of the duct. The present results show that this spanwise limitation of the oscillating region strongly diminishes the drag reduction potential of the control technique. We propose a simple model that allows estimating the achievable drag reduction rates in duct flows as a function of the width of the duct and the spanwise extent of the controlled region.
\end{abstract}

Keywords DNS · control · oscillating walls $\cdot$ secondary flow $\cdot$ duct flow

\section{Introduction}

Flow control with the goal of reducing turbulent skin-friction drag at fluid-solid interfaces is an active fundamental research area, motivated by its potential for significant energy savings in the transport sector. Among the control strategies currently under development, active techniques introduce energy into the flow through actuators and are intensively investigated, because of the sizeable turbulent skin-friction drag reduction that they yield. Particularly interesting are active predetermined techniques, whose control action is applied regardless of the state of the flow, with the advantage of moderate complexity, as no sensors and only relatively large-scale actuators are required. A very successful predetermined approach is the

$\bowtie \quad$ S. Straub

steffen.straub@kit.edu

1 Institute of Fluid Mechanics, Karlsruhe Institute of Technology, 76131 Karlsruhe, Germany

2 Linné FLOW Centre and Swedish e-Science Research Centre (SeRC),

KTH Mechanics, Royal Institute of Technology, 10044 Stockholm, Sweden 
near-wall turbulence modification through large-scale, in-plane spanwise forcing created by wall motions $[1,2]$.

Spatially homogeneous spanwise wall oscillations [3] are the simplest, although not the most efficient, among these strategies and are the basis onto which more complex and better performing wall-forcing techniques are designed, such as the streamwise-travelling waves of spanwise velocity [4] or the rotating and oscillating discs [5, 6]. Because of this, spanwis/e wall oscillation has been the framework of choice to take on the unanswered questions in the field of turbulent drag reduction, such as the Reynolds-number scaling of drag reduction [7$10]$, its complex underlying physics [11-14] or its very challenging practical implementation $[15,16]$.

In spite of its simplicity, spanwise oscillations have been investigated mostly numerically through direct numerical simulation (DNS), while only few laboratory experiments have been performed in boundary layers [16-20], pipes [21, 22] or duct flows [15]. The available evidence shows that the drag reduction rate, defined as control-induced change of skin-friction relative to the uncontrolled flow, is smaller in laboratory experiments compared to similar numerical investigations, specifically for those experiments which rely on integral friction measurements.

A first reason for the discrepancy is the fact that laboratory experiments are usually performed at slightly higher values of Reynolds number $R e$ than the simulation they are compared to. Typical values for the friction Reynolds number $R e_{\tau}=u_{\tau} h / v$, dfefined by the friction velocity $u_{\tau}=\sqrt{\tau_{w} / \rho}$ obtained from the wall-shear stress $\tau_{w}$ and density $\rho$, the channel half height $h$ and the kinematic viscosity $v$, are $R e_{\tau}=600$ and $R e_{\tau}=200$ for laboratory experiments and simulations respectively. Combined with the known degradation of the achievable drag reduction for increasing values of Reynolds number $R e$ [8], which can be severe at low values of $R e$, this can be identified as one source for deviations. Skote [23], for instance, performed a DNS of a turbulent boundary layer modified by spanwise oscillations at $\operatorname{Re}_{\theta}=500$ (where $\operatorname{Re}_{\theta}$ is the Reynolds number based on momentum thickness) and compared the results against the laboratory experiment performed by Ricco and Wu [19] at the same oscillation conditions but higher $R e_{\theta}=1400$. With an analysis of available literature data, Skote showed that the differences in the results $(23 \%$ drag reduction rate was measured in the experiment and $28.6 \%$ was obtained in the simulation) could be almost entirely attributed to the different values of $R e$.

However, different values of $R e$ are not the only possible cause of discrepancies between numerical and laboratory experiments. An example is the experiment and companion numerical simulation performed by Gatti et al. [15], who measured the pressure drop in a turbulent duct flow at $R e_{\tau}=210$ and showed that the oscillations of a limited portion of the duct walls yield a drag reduction rate of $2.4 \%$, to be compared with the $10 \%$ measured via DNS at the same value of $R e$ and (suboptimal) oscillation conditions. Such discrepancies are usually ascribed to the inhomogeneous distribution of spanwise wall velocity, necessarily present in experiments, compared to the ideal setting of numerical simulation, in particular of turbulent channels, in which the oscillating portion of the wall extends indefinitely. An additional difference between experiments and numerics is the streamwise spatial transient along which the friction slowly decreases from its unperturbed level down to the drag-reduced level induced by the control. This transient, which is typically not considered in numerical studies but unavoidably present in experiments, can impact severely on the control performance and has therefore been extensively studied (see, for instance, [15, 23-25]).

The present work aims at addressing the effect of inhomogeneous distribution of spanwise wall velocity on the achievable drag reduction via direct numerical simulation of turbulent duct flows, in which either the whole wall or a spanwise-limited portion thereof is 
Table 1 Geometrical parameters and resolution of the uncontrolled channel and duct reference cases for the present study

\begin{tabular}{cccccccccccc}
\hline & $L$ & $W$ & $H$ & $\mathrm{AR}$ & $\Delta x_{\max }^{+}$ & $\Delta y_{\max }^{+}$ & $\Delta y_{\min }^{+}$ & $y_{10}^{+}$ & $\Delta z_{\max }^{+}$ & $\Delta z_{\min }^{+}$ & $z_{10}^{+}$ \\
\hline $\mathrm{c}: 0$ & $4 \pi h$ & $2 \pi h$ & $2 h$ & $\pi$ & 14.8 & 7.3 & 0.2 & 7.5 & 7.4 & 2.3 & $/$ \\
$\mathrm{d}: 0$ & $12.5 h$ & $6 h$ & $2 h$ & 3 & 14.7 & 7.3 & 0.4 & 9.9 & 7.5 & 0.4 & 9.9 \\
\hline
\end{tabular}

periodically oscillated. We show that a limited spanwise extent of an oscillating wall and the related generation of secondary motions strongly diminishes the drag reduction potential of infinitely-large oscillating walls. This factor significantly contributes to the differences observed between experimental and numerical studies. In order to estimate the achievable drag reduction potential within a duct geometry, we present a simple model based on the width of the duct and the spanwise extent of the controlled region.

\section{Methodology}

DNS of turbulent channel and duct flows at constant flow rate with aspect ratio $A R=3$ (where $A R$ is defined as the ratio between the duct width and its height) are performed with a highly parallelized code using the spectral-element method, Nek5000 [26].

The spectral-element code is more computationally expensive than a classic pseudospectral code for performing direct numerical simulations of turbulent channels of a given size. However, it allows to combine the geometrical flexibility of finite-element methods with the accuracy of spectral methods, and therefore it is the method of choice in the present study due to the moderate geometrical complexity introduced by the side walls. In the spectral-element method the domain is divided into a number of elements similar to the finite-element method. Within each element the unknowns are represented as a polynomial of a certain order [27]. In our simulations, the velocity field is represented by an $N$-th order polynomial and the pressure field by an $N$-2-th order polynomial, which is the so-called $\mathbb{P}_{N}-\mathbb{P}_{N-2}$ formulation. The streamwise pressure gradient required to impose a constant flow rate is determined implicitly during each time step [28].

The numerical solver Nek5000 is validated for a standard turbulent channel flow at $R e_{b}=u_{b} h / v=2800$ [29] and the corresponding implementation of the wall oscillation is validated against in-house [30] DNS data obtained with an established pseudo-spectral DNS code as shown in the Appendix. An uncontrolled duct flow at $R e_{b}=2581$ serves as reference case for the present duct-flow investigation. This value is chosen such that the local Reynolds number at the centreplane $R e_{b, z 0}=\bar{u}_{b, z 0} h / v \approx 2800$ is comparable to the channel simulations, with $\bar{u}_{b, z 0}$ being the averaged streamwise velocity in the vertical centreplane at $z=0[31]$.

The geometrical dimensions, streamwise length $L$, spanwise width $W$ and wall-normal height $H$, and the mesh parameters are summarized in Table 1 . Non-dimensionalization with the kinematic viscosity $v$ and the friction velocity $u_{\tau}$ is denoted by the superscript ${ }^{+}$. The friction velocity of the duct is based on the global wall-shear stress, i.e. averaged over all four walls. The number of elements in streamwise, wall-normal and spanwise direction is 32,16 and 36, respectively. The Polynomial order is 7 , which gives 8 points per element and direction. In the streamwise direction, the spectral elements are distributed equidistantly. In the wall-normal direction, the element location is defined by $\sin (\pi / 2 \cdot \zeta)+1$, where the variable $\zeta$ represents 17 equally-spaced points in the interval $[-1,1]$. The sizes of the first 
Table 2 Different control parameters for channel and duct cases

\begin{tabular}{llllll}
\hline & $\mathrm{c}: 12$ & $\mathrm{c}: 4.5$ & $\mathrm{~d}: 12$ & $\mathrm{~d}: 4.5$ & $\mathrm{p}: 4.5$ \\
\hline$A^{+}$ & 12 & 4.5 & 12 & 4.5 & 4.5 \\
moving region $z / h$ & {$[-3,3]$} & {$[-3,3]$} & {$[-3,3]$} & {$[-3,3]$} & {$[-1.5,1.5]$} \\
\hline
\end{tabular}

two elements next to the wall are manually adjusted to be $\Delta y / h=0.035$ and $\Delta y / h=0.086$ to avoid a too strong clustering. In the spanwise direction, the distribution of elements is the same as in wall-normal direction when approaching the side walls at $z / h \in[-3,-2]$ and $z / h \in[2,3]$. In between, the elements are distributed equidistantly. The points inside the spectral elements follow the Gauss-Lobatto-Legendre (GLL) distribution. The subscripts min, $\max$ and 10 in Table 1 denote the minimum and maximum distance between two neighbouring grid points and the distance of the 10th point from the wall.

The uncontrolled channel case is denoted by c:0, where c abbreviates channel and the number stands for the inner-scaled amplitude of wall velocity, which is 0 for the uncontrolled case. Likewise, the uncontrolled duct case is denoted by $\mathrm{d}: 0$. This naming convention is used henceforth. In addition to the two uncontrolled cases, five controlled configurations were simulated: two channel and three duct flows.

The periodic oscillation of the upper and lower walls is enforced by prescribing the spanwise velocity of the fluid as:

$$
w(y / h=0)=w(y / h=2)=A \sin \left(\frac{2 \pi}{T} t\right)
$$

with $A$ being the maximum wall velocity or amplitude and $T$ the oscillation period. The oscillation period is kept constant in all cases and equals $T^{+}=125$, which is found by Quadrio and Ricco [3] to be the value that maximizes the achievable drag reduction. The amplitude is varied as $A^{+} \in\{0,4.5,12\}$ based on the uncontrolled friction velocity. The five controlled cases and their corresponding control parameters are summarized in Table 2. In Fig. 1 (a) a schematic drawing illustrates the setup of cases d:4.5 and d:12. The spanwise

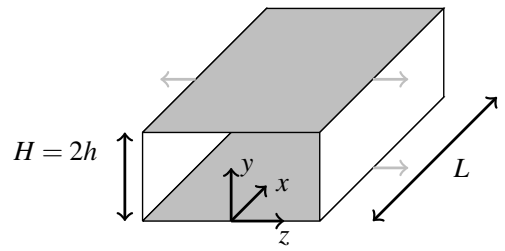

(a)

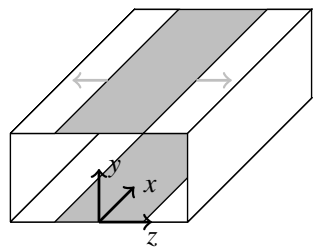

(b)

Fig. 1 Schematic drawings of (a) controlled duct cases d:4.5 and d:12 and (b) case p:4.5 [32]

wall motion is set to zero at the side walls. To avoid numerical problems, a continuouslydifferentiable smooth ramp function is used over a small distance $\Delta \lambda$ in the transition region between zero wall velocity and the oscillation velocity. This smooth ramp function is defined 
as:

$$
S(\xi)= \begin{cases}0, & \xi \leq 0, \\ \frac{1}{1+\exp (1 /(\xi-1)+1 / \xi)}, & 0<\xi<1, \\ 1, & \xi \geq 1 .\end{cases}
$$

The smooth ramp function is applied to both spanwise boundaries of the controlled region and is given as follows for cases $\mathrm{d}: 4.5$ and $\mathrm{d}: 12$

$$
\lambda(z)=S\left(\frac{z / h+3}{\Delta \lambda}\right) \cdot S\left(\frac{-z / h+3}{\Delta \lambda}\right)
$$

where the factor $\Delta \lambda / h=0.035$ determines the width of the ramp.

Due to the representation of this analytical function as a Lagrangian polynomial through the GLL points within the individual elements, this steep gradient cannot be perfectly captured by the polynomial and an error similar to the Gibbs phenomenon is introduced. An overshoot of $6 \%$ for the ramp is found, which implies that the peak of the polynomial is $6 \%$ larger than the actual value. It has been confirmed that the resulting wall-shear stress is not significantly influenced by the width of this transition region observed in simulations with a value of $\Delta \lambda$ six times larger. For $\mathrm{d}: 4.5$ and $\mathrm{d}: 12$ the ramp function is located directly at the side walls and for case p:4.5 (partial control) it is located at $z / h=-1.5$ and $z / h=1.5$, limiting the applied control technique only to the inner region. This is depicted in Fig. 1 (b). For both, channel and duct, the instantaneous quantities are decomposed into mean $(\bar{\sim})$ and fluctuating values $\left(\sim^{\prime}\right)$. Previous studies on oscillating walls have employed a triple decomposition $[13,33]$ in such a way that the harmonic oscillation of the wall is removed from the fluctuating values. In the present work we did not apply such a phase-resolved approach since we aim at extracting global effects in the duct flow, which are strongly connected to the generated secondary motions.

All quantities are averaged along the statistically homogeneous directions, resulting in a slightly different averaging for duct and channels. Results of the channel cases are averaged over time, streamwise and spanwise direction. Results of the duct cases are averaged over time and streamwise direction only, since the spanwise direction is inhomogeneous. In addition, four-fold symmetry conditions in the duct are exploited to further improve the statistics. Nevertheless, the averaging time in the duct simulations needs to be significantly longer than in the channel to compensate for the absence of averaging in spanwise direction. Statistical averaging starts after the initial transient has passed (i.e. more than 100 convective time units later [34]) and statistics are collected for at least 300 convective time units for the channel flows [35] and 2000 time units for the duct flows [36].

\section{Results \& Discussion}

A compact representation of the four investigated duct cases is used for showing the different features of each case and comparing them. Figure 2 shows the partitioning of the following 2D colour plots, where the cross section of the duct is separated into four quarters each representing one of the four duct cases. Note that one quarter is sufficient to represent each case due to four-fold symmetry in the duct. All results are scaled in outer units, i.e. with half-height $h$ and bulk velocity $u_{b}$ unless otherwise stated. 


\begin{tabular}{|l|l|}
\multicolumn{1}{c|}{$y$} \\
\hline $\mathrm{d}: 4.5$ & $\mathrm{~d}: 0$ \\
\hline $\mathrm{d}: 12$ & $\mathrm{p}: 4.5$ \\
\hline
\end{tabular}

Fig. 2 Partitioning of 2D contour plots

\subsection{Turbulence Statistics}

Figure 3 (a) shows the mean streamwise velocity $\bar{u}$ of the uncontrolled duct case and the three controlled cases, partitioned as described above. The uncontrolled and controlled cases

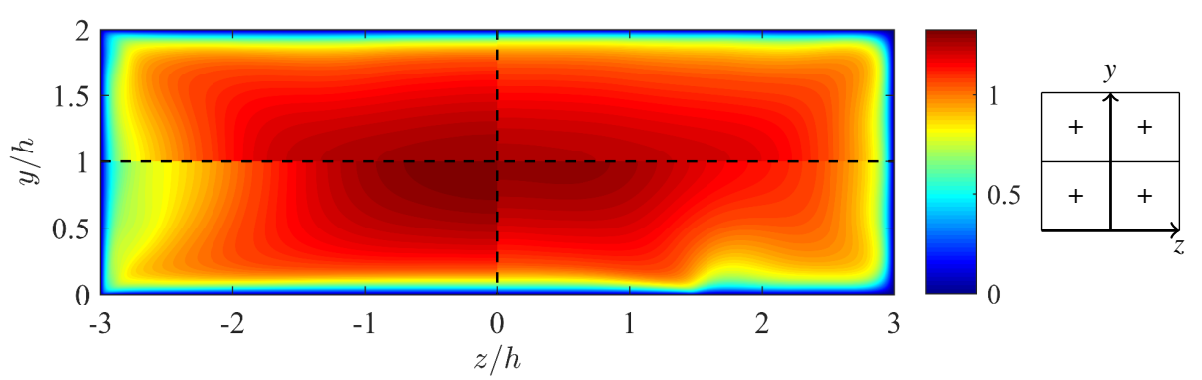

(a)

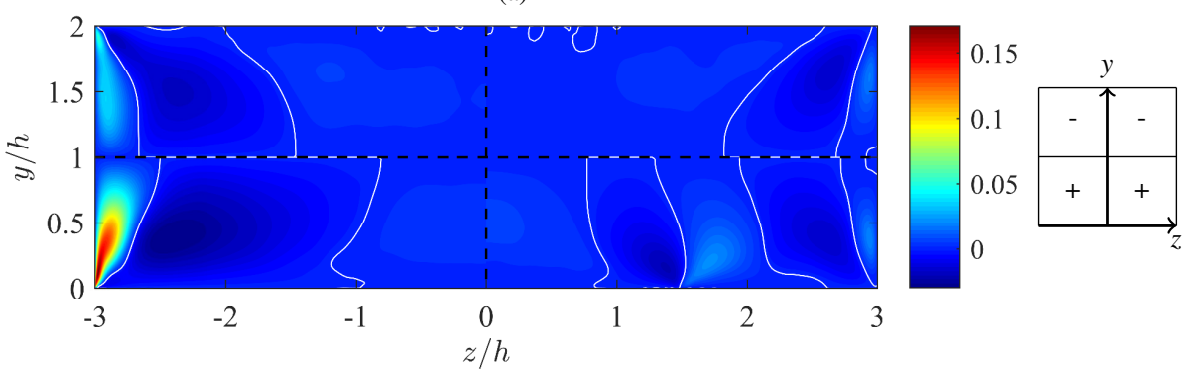

(b)

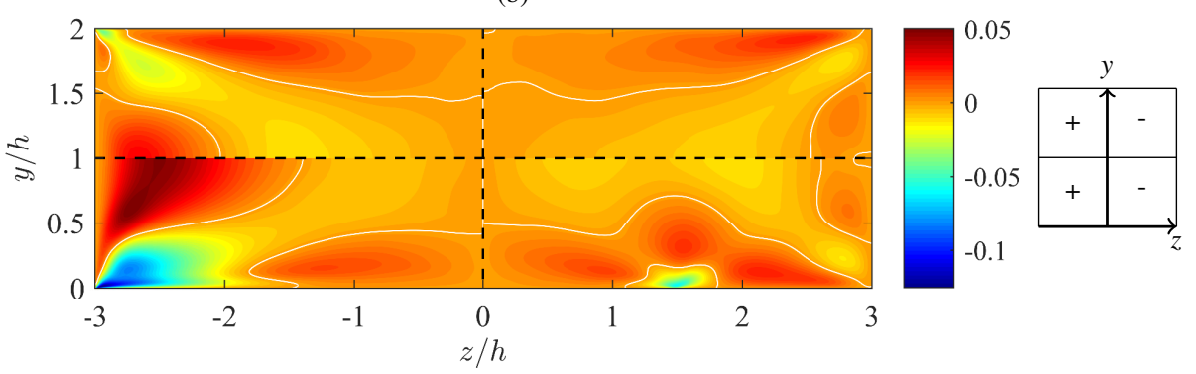

(c)

Fig. 3 Mean velocities in (a) streamwise direction, (b) wall-normal (y-direction) and (c) spanwise direction of the four duct cases, scaled in outer units and partitioned as shown in Fig. 2. Sketch on the right indicates quarters that are multiplied by -1 with "-_" to facilitate comparison [32]. 
differ mostly along the side walls for $\mathrm{d}: 4.5$ and $\mathrm{d}: 12$ or around the lateral end of the control section for p:4.5. There, significant alterations of the mean velocities can be observed. For $\mathrm{d}: 4.5$ and $\mathrm{d}: 12$ we find regions of decreased mean streamwise velocity at the side wall compared to d:0. The same occurs for p:4.5 at $z / h \approx 1.7$, i.e. in the vicinity of the edges of the control section.

Closer inspection reveals a difference in the corner regions of $\mathrm{d}: 0, \mathrm{~d}: 4.5$ and $\mathrm{d}: 12$. The control-induced secondary motions enhance the displacement of high-speed fluid from the duct core towards the corners. As a result, the portion of the horizontal wall which exhibits a velocity gradient $\partial \bar{u} / \partial y$ comparable to (or larger than) what achieved at the duct centreplane gets closer to the corners compared to the uncontrolled flow. For $\mathrm{p}: 4.5$, there is also a region of increased $\partial \bar{u} / \partial y$ at the edge of the control section.

A more quantitative assessment of the effect of the side walls on the mean streamwise velocity profile is possible by comparing the profiles of $\bar{u}^{+}$in the centreplane of the duct, $z=$ 0, shown in Fig. 4. Due to the inhomogeneity in spanwise direction, a local friction velocity

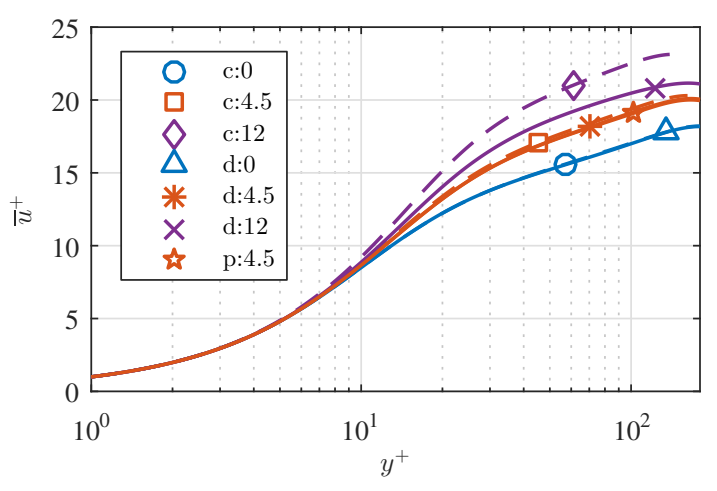

Fig. 4 Comparison of inner-scaled mean streamwise velocity profiles at $z=0$ for duct and channel cases. Blue lines correspond to uncontrolled flows, red ones to $A^{+}=4.5$ and purple ones to $A^{+}=12$.

$\overline{u_{\tau}}(z)$ is defined as $\sqrt{\overline{\tau_{w}}(z) / \rho}$ with the local wall-shear stress $\overline{\tau_{w}}(z=0)$. Thus, the local friction velocity $\overline{u_{\tau}}(z=0)$ is used for the inner scaling of the duct cases at this position. This plot additionally includes the corresponding channel cases. The mean streamwise velocity profile of the uncontrolled duct case d:0 coincides with the uncontrolled channel case c:0 even at this low aspect ratio. Moreover, the controlled cases d:4.5 and p:4.5 also agree well with their respective channel case c:4.5. However, for larger oscillation amplitude, the mean streamwise velocity in the controlled channel (c:12) is $9 \%$ higher than in the centre of the controlled duct (d:12).

The mean wall-normal and spanwise velocities of each duct case are shown in Figs. 3 (b) and (c), respectively. To facilitate comparison, the two upper quarters in Fig. 3 (b) are multiplied with -1 , as well as the two right quarters in Fig. 3 (c). Additionally, white contour lines of zero velocity are includfed. Non-negligible mean wall-normal velocity is observed at the spanwise boundaries of the controlled region. It can be seen that at those positions fluid, in temporal average, moves away from the oscillating walls. This is especially apparent for d:12 with $\bar{v}_{\max } / u_{b}=0.17$ but also visible for $\mathrm{d}: 4.5$ with $\bar{v}_{\max } / u_{b}=0.05$ and $\mathrm{p}: 4.5$ with $\bar{v}_{\max } / u_{b}=0.02$. As this motion carries fluid with low mean streamwise velocity from the near-wall region towards the core, it can directly be linked to the regions of reduced streamwise mean velocity observed in Fig. 3 (a). The presence of those relatively large mean flow velocities in the wall-normal direction observed for the oscillating wall suggests 
the introduction of secondary motions in the duct cross-section which are much stronger than the well-known secondary motions of Prandtl's second kind in duct flows.

The corresponding mean flow in the spanwise direction is shown in Fig. 3 (c). Two regions on top of each other where fluid is moving in different directions are found in all three controlled cases. This behaviour is most clearly observed in the corner of case d:12, where fluid is moving in the negative spanwise direction close to the wall (blue region) and in the positive spanwise direction (red region) at $z / h \approx-2.5$. Those generated secondary motions are discussed further in the following subsection.

The root mean square (RMS) $u_{r m s}=\sqrt{\overline{u^{\prime} u^{\prime}}}$ of the streamwise velocity fluctuations are shown in Fig. 5 (a). It can clearly be seen that the streamwise velocity fluctuations, non-

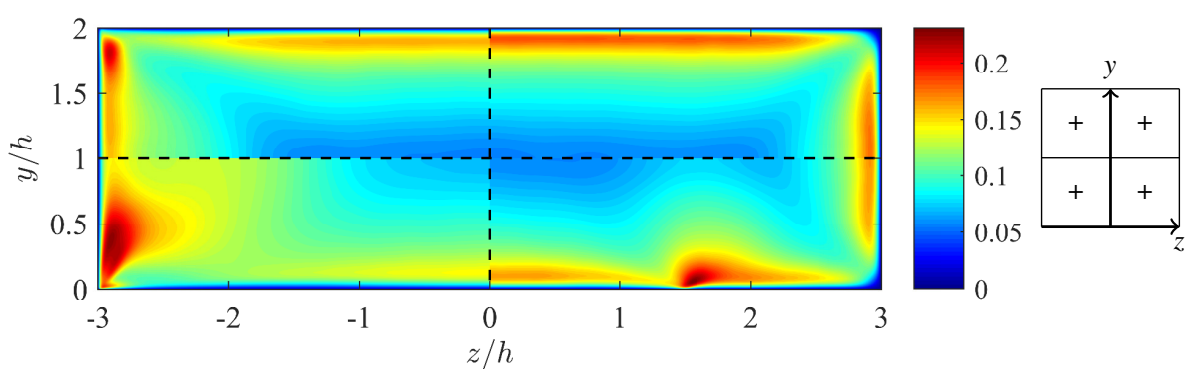

(a)

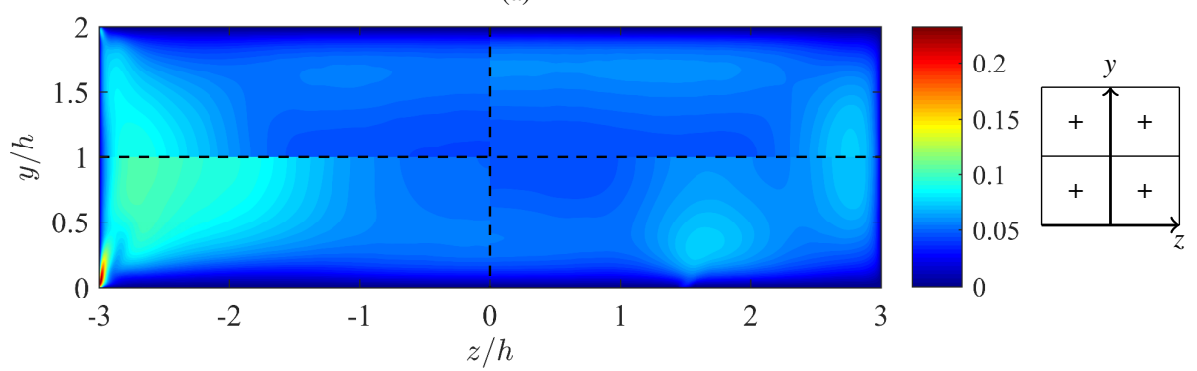

(b)

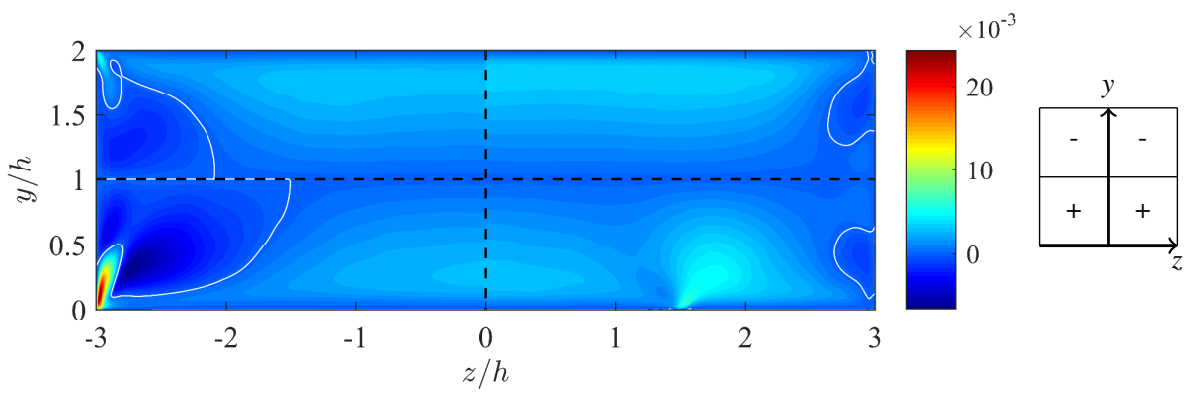

(c)

Fig. 5 RMS of the (a) streamwise velocity fluctuations, (b) wall-normal velocity fluctuations and (c) Reynolds shear stress $-\overline{u^{\prime} v^{\prime}}$ of the four duct cases, scaled in outer units and partitioned as shown in Figure 2. Sketch on the right indicates quarters that are multiplied by -1 with "-_" to facilitate comparison [32]. 
dimensionalized in outer units, are diminished above the oscillation regions. This corresponds to observations made in turbulent channel flows over oscillating walls at constant flow rate [13]. At the same time an increase of $u_{r m s}$ next to the oscillating region, i.e. along the vertical side walls in cases $\mathrm{d}: 4.5$ and $\mathrm{d}: 12$ or for $z / h>1.5$ in case of $\mathrm{p}: 4.5$, is clearly visible. These regions of strongly elevated $u_{r m s}$ in the controlled duct flows are directly related to the spanwise wall motion itself.

The spanwise oscillation periodically pushes fluid towards the side walls and pulls it away again. Pushing fluid towards the side walls generates an upwash motion at the edge of the oscillation region. Those events are characterized by relatively slow-moving near-wall fluid moving upwards in a region of faster motion which we refer to as a large-scale ejection event. During the other half of the oscillation cycle a corresponding large-scale sweep event is produced. The presence of those events can naturally also be seen in in Fig. 5 (b) for $v_{r m s}$ which reveals increased fluctuation levels in the same regions of the duct as for $u_{r m s}$.

Figure 5 (c) shows the correlation of the streamwise and wall-normal velocity fluctuations, $-\overline{u^{\prime} v^{\prime}}$. This quantity, the Reynolds shear stress, can be directly related to skin-friction drag in turbulent channel flows [37] and is therefore expected to be reduced if drag-reducing control is successfully applied. For the present results, it can indeed be seen that $-\overline{u^{\prime} v^{\prime}}$ is reduced above the oscillation regions. However, its strong increase at the spanwise edges of the controlled region (due to the generated large-scale ejection and sweep events) indicates a strong negative impact on the achievable drag reduction in duct flows.

\subsection{Secondary Flow}

As described above, the inhomogeneous spanwise wall motion induces additional secondary vortices in the duct flow. The formation of those secondary vortices results from the periodic large-scale sweep and ejection events which do not cancel each other in a temporal average. In contrast to the secondary motions of Prandtl's second kind, those vortical motions are also generated in laminar channels with and without mean streamwise velocity, when part of the wall oscillates in the spanwise direction. For instance, Fig. 6 shows the mean wallnormal velocity of a laminar channel flow driven at the same flow rate as the other cases. As

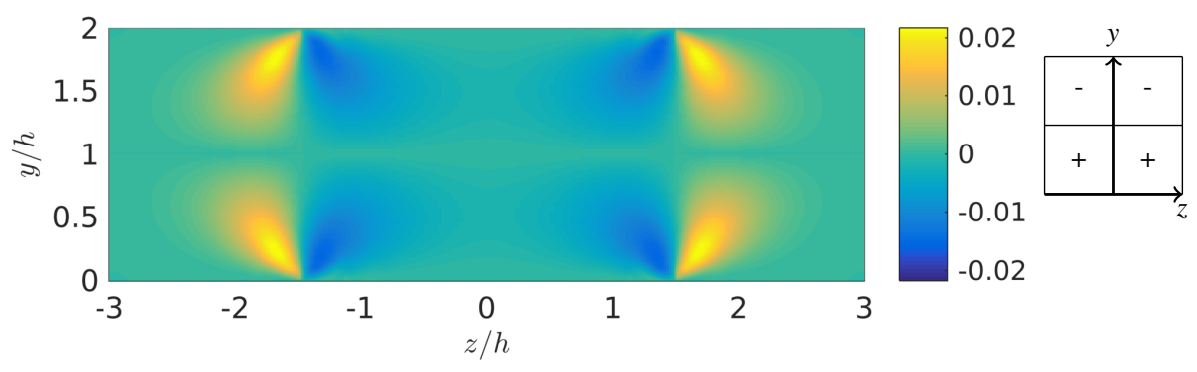

Fig. 6 Mean wall-normal velocity in a spanwise-limited controlled laminar channel flow in outer units with $A^{+}=4.5, T^{+}=125$. The controlled region is between $z / h=-1.5$ and $z / h=1.5$.

in p:4.5 the controlled region is located between $z / h=-1.5$ and $z / h=1.5$. The persistence of these vortical motions in the mean flow field, due to the asymmetric behaviour of the flow at the edges of the controlled region in the two oscillation semi-cycles, can be explained by 
considering the bottom half of the channel at the location $z / h=-1.5$. During the leftward motion of the wall, flow with high spanwise momentum is ejected away from the wall in the upward outward direction. On the other hand, during the rightward motion, flow with low spanwise momentum from the core of the channel is pulled downwards towards the edge of the controlled region. The downward and upward motions occur at different locations, intensities and spatial extent. In fact, the ejection of intense spanwise momentum occurs in a more confined region compared to the sweep of low-spanwise-velocity fluid towards the wall. This is in agreement with the observations in the turbulent duct flow (compare Fig. 3 (b)).

The strength of the secondary motion can be evaluated in terms of its kinetic energy [36], which is defined as:

$$
K=\frac{1}{2}\left(\bar{v}^{2}+\bar{w}^{2}\right),
$$

where $\bar{v}$ and $\bar{w}$ are the mean wall-normal and spanwise velocities scaled in outer units, respectively. Two-dimensional colour plots of $K$ are presented in Fig. 7 together with equally spaced streamlines. The partitioning is again the same as described above. Each case uses a

(a)

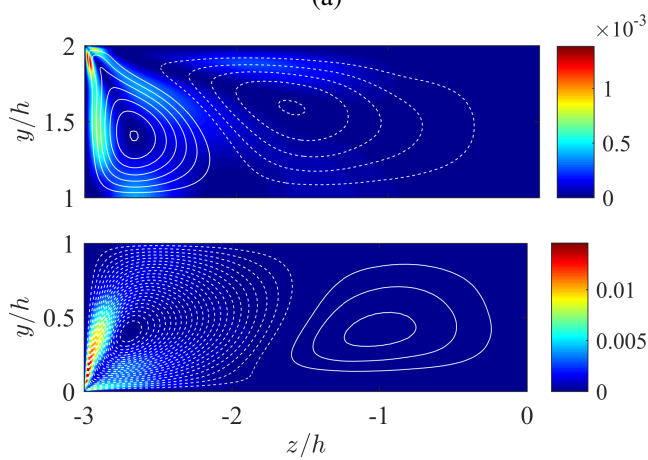

(c) (b)

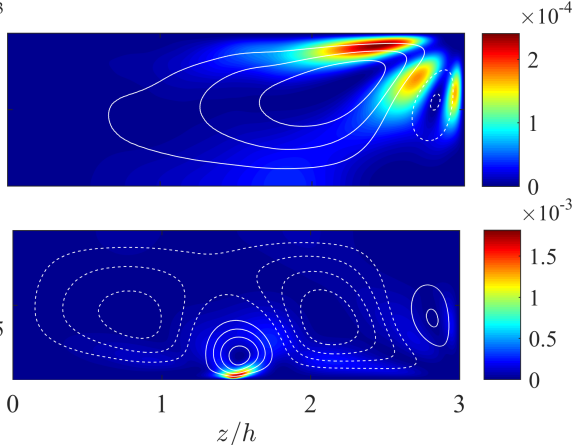

(d)

Fig. 7 Secondary flow field of (a) d:4.5, (b) d:0, (c) d:12 and (d) p:4.5 in different corners according to Fig. 2. The kinetic energy of the secondary flow $K$ is shown, together with equally-spaced streamlines. Dashed lines indicate clockwise rotation and continuous lines anticlockwise rotation [32].

different scaling for $K$ but the spacing between two adjacent streamlines is kept constant for all cases.

It is clearly visible that the secondary motions induced by the inhomogeneous wall oscillation carry a kinetic energy that is one or two orders of magnitude stronger than the secondary motions of Prandtl's second kind observed for the uncontrolled duct flow d:0. The case d: 12 produces the strongest secondary vortices with $K_{\max }=1.5 \times 10^{-2}$ while d:4.5 and p:4.5 share roughly the same kinetic energy of the secondary flow: $K_{\max }=1.4 \times 10^{-3}$ and $K_{\max }=1.8 \times 10^{-3}$. The density of the streamlines is a measure of the flow rate of the in-plane motion while the plotting style (dashed or continuous lines) indicates the corresponding direction of rotation. It can be seen that the rotational direction of the secondary corner vortex pair relative to the duct geometry is the same for all cases. Compared to d:0, the wall oscillation in $\mathrm{d}: 4.5$ and $\mathrm{d}: 12$ strongly enhances the secondary-flow motion that carries fluid from the corner of the oscillating wall along the side walls toward the duct centre 
and high-speed fluid from the duct centre towards the corner region. For the partial wall oscillation in p:4.5 the anticlockwise vortex generated at the edge of the oscillating section is flanked by two larger counter-rotating vortices while a second vortex with anticlockwise rotational direction is located in the duct corner. This vortex, which resides along the vertical wall, is very similar to the one observed in $\mathrm{d}: 0$ at the same location. A closer inspection reveals that it is slightly enhanced compared to the uncontrolled case.

\subsection{Drag Reduction}

In order to compare the performance of the controlled channel cases and the controlled duct cases, the global drag reduction rate is defined as:

$$
D R=\frac{C_{f, 0}-C_{f}}{C_{f, 0}},
$$

with $C_{f, 0}=2 \tau_{w, 0} /\left(\rho u_{b}^{2}\right)$ and $C_{f}=2 \tau_{w, c} /\left(\rho u_{b}^{2}\right)$ being the skin-friction coefficient of the uncontrolled and controlled cases, respectively. We evaluate the uncertainty of $D R$ using the method described in Oliver et al. [38] to quantify the sampling error of the obtained correlated data. Following their procedure, the empirical standard deviation of the sampling error is calculated for controlled and uncontrolled cases. Propagation of errors yields the empirical standard deviation of $D R$ as

$$
\Delta D R=\frac{C_{f}}{C_{f, 0}} \sqrt{\left(\frac{\Delta C_{f, 0}}{C_{f, 0}}\right)^{2}+\left(\frac{\Delta C_{f}}{C_{f}}\right)^{2}}
$$

under the assumption of statistically independent errors [8]. This is extended to a $95 \%$ confidence level by taking $\Delta D R$ twice as the standard uncertainty of $D R$. For all three controlled duct cases the uncertainty is about $1 \%$ or less.

The global drag reduction rate is displayed in Fig. 8 for all five controlled cases. The

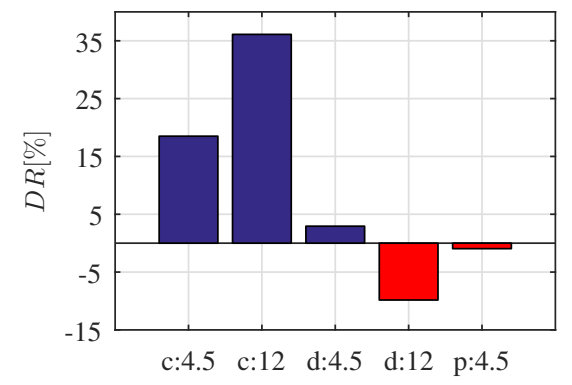

Fig. 8 Comparison of global drag reduction rate [32]

control achieves lower, sometimes negative, values of drag reduction in ducts compared to channels. The worst performance in terms of drag reduction is observed for $\mathrm{d}: 12$ with a drag increase of $\approx 10 \%$. This loss of drag reducing performance is in agreement with the observed large-scale sweep and ejection events at the edges of the controlled regions discussed before.

The global drag reduction rate is split into the contributions from the horizontal walls $D R_{h o r}$ and the side walls $D R_{v e r}$ in order to further analyse the large differences between 
Table 3 Levels of drag reduction rate averaged over the four walls $(D R)$, over the horizontal walls $\left(D R_{h o r}\right)$ and the vertical walls $\left(D R_{\text {ver }}\right)$

\begin{tabular}{|c|c|c|c|c|c|}
\hline & c: 12 & $\mathrm{c}: 4.5$ & $\mathrm{~d}: 4.5$ & $\mathrm{~d}: 12$ & $\mathrm{p}: 4.5$ \\
\hline$D R \quad[\%]$ & 36.1 & 18.5 & 2.9 & -9.8 & -1.0 \\
\hline$D R_{\text {hor }}[\%]$ & 36.1 & 18.5 & 5.0 & -9.0 & -2.4 \\
\hline$D R_{\text {ver }}[\%]$ & I & I & -4.3 & -13.0 & 4.2 \\
\hline
\end{tabular}

controlled duct and channel simulations. This is given in Table 3. The local drag reduction rate at the horizontal walls exhibits the same trends as the global drag reduction rate. For $\mathrm{d}: 4.5$ and $\mathrm{d}: 12$ the skin-friction is largely increased at the side walls, while p:4.5 interestingly reveals drag reduction at this location.

The local wall-shear stress distribution on the horizontal and vertical walls is displayed in Fig. 9. This quantity, averaged in the streamwise direction and in time, is normalized by its local value in the vertical centreplane (at $z=0$ ) of the corresponding uncontrolled case $\bar{\tau}_{w, 0} z 0$. Drag reduction for the channel cases is clearly seen by the downward shift of the

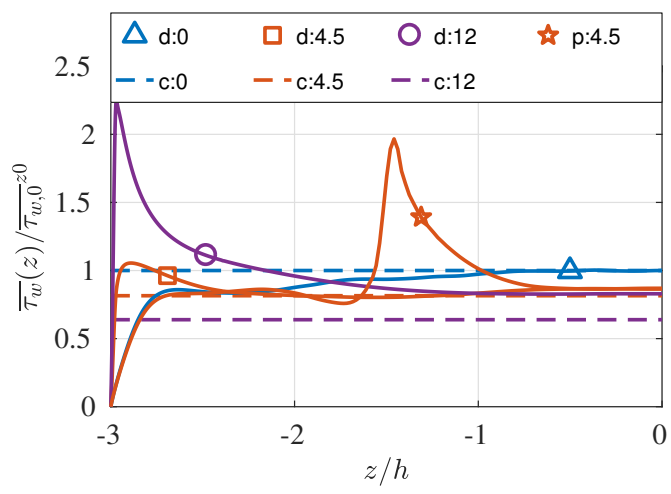

(a)

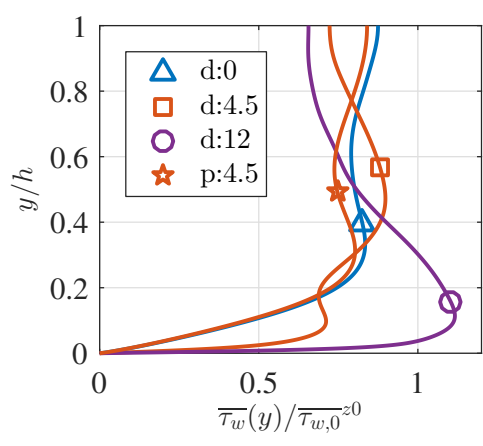

(b)

Fig. 9 Mean wall-shear stress along (a) horizontal wall and (b) vertical wall normalized with the wall-shear stress of the corresponding uncontrolled flow in the centreplane

respective dashed lines with c:12 being lower than c:4.5 and c:0 being unity. Regarding the duct cases, the two configurations with $A^{+}=4.5$ show approximately the same skin-friction reduction as the channel with same $A^{+}$in the centreplane region. The $\mathrm{d}: 4.5$ case shows slightly increased wall-shear stress close to the side wall compared to the uncontrolled duct case, whereas the partially-controlled case p:4.5 exhibits a peak of almost $2 \overline{\tau_{w, 0}} z 0$ at the edge of the oscillating region. Note that in the p: 4.5 case, the uncontrolled region $-3<z / h<-2$ follows the same wall-shear stress curve as the baseline case d:0. The low wall-shear stress level of the channel case c:12 is not achieved in the centreplane of the corresponding duct configuration d:12. However, a local wall-shear stress reduction compared to d:0 is visible, where the centreplane value is approximately the one obtained in the cases with $A^{+}=4.5$. On the other hand, the large wall-shear stress peak near the side walls clearly outweighs this local benefit. The local wall-shear stress maxima observed in the p:4.5 and d:12 cases 
are directly related to the introduced secondary motions that on average transport fluid with relatively large streamwise velocity into those near-wall regions.

The local wall-shear stress distribution along the side walls $\overline{\tau_{w}}(y)$, normalized with the local wall-shear stress at the centreplane of the duct (at $z=0$ ) is given in Fig. 9 (b). The uncontrolled case shows a first peak at $y / h \approx 0.3$ and a second peak at the vertical centre of the duct at $y / h=1$. A very similar behaviour is shown by the controlled case with moving walls limited to the inner region of the horizontal walls, $\mathrm{p}: 4.5$. However this curve exhibits slightly lower values, a fact that explains the drag reduction rate mentioned in Table 3 . This behaviour is connected with the slightly increased strength of the secondary motion located along the vertical side wall, which is known to impact the wall-shear stress distribution in uncontrolled duct flows. The behaviour observed in the d:4.5 and d: 12 cases is also closely related to the corresponding secondary motions. In those cases the corner region is dominated by the secondary vortices that arise from the large-scale sweep and ejection events. Within each oscillation, fast-moving fluid is driven from the centre towards the side wall in half of the oscillation period. This impingement generates the increased wall-shear stress levels visible in Fig. 9 (b).

A trace of the periodic oscillation is also visible in the local RMS of the wall-shear stress on the lower duct wall as depicted in Fig. 10, where strong increases of $\tau_{w, r m s}(z)$ are visible at the edges of the oscillating regions. Besides these locally-increased values, the controlled

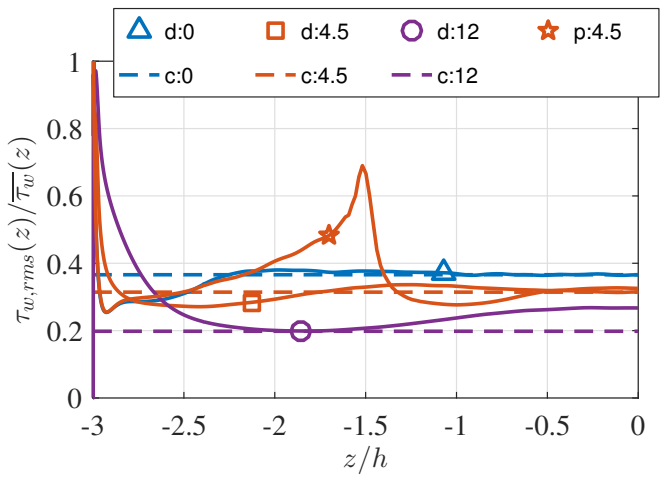

Fig. 10 Fluctuating wall-shear stress distributions along horizontal wall, normalized with the local mean wall-shear stress values

duct cases with $A^{+}=4.5$ show remarkably good agreement with the corresponding channel flow curves in the middle of the controlled region and with the values of the uncontrolled duct in the uncontrolled region in the p:4.5 case. The impossibility of achieving the wallshear stress levels from the c: 12 case with the corresponding controlled-duct d:12 observed in Fig. 9 is also reflected in the RMS results shown in Fig 10, where only local agreement in the region around $z / h \approx-1.8$ is observed. Nevertheless, the good agreement in first- and second-order statistics between the controlled duct flow and the corresponding controlled channel flow with $A^{+}=4.5$ in some locations, and the agreement with the uncontrolled duct flow in others, suggests the possibility of developing a model for the estimation of achievable wall-shear stress reduction in partially-controlled duct flows. Such a model is described in the next section. 


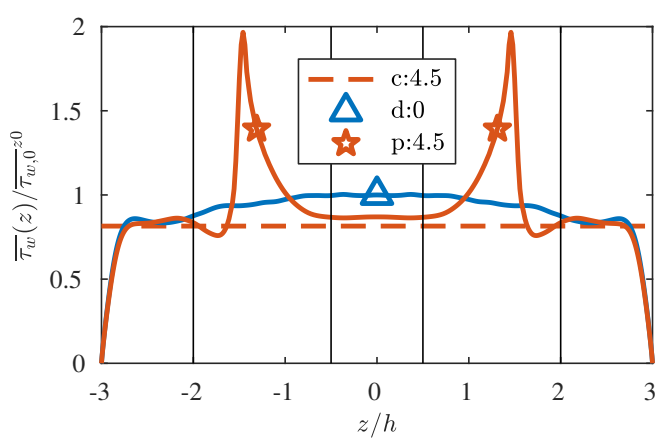

Fig. 11 Decomposition of $\overline{\tau_{w}}(z)$ along the horizontal wall for the partially-controlled case $\mathrm{p}: 4.5$

Table 4 Regionally-averaged wall-shear stresses scaled by the uncontrolled value in the centre of the duct and channel respectively

\begin{tabular}{lrrr}
\hline & ${\overline{\tau_{w}}}^{0} /{\overline{\tau_{w, 0}}}^{z 0}$ & ${\overline{\tau_{w}}}^{p n} /{\overline{\tau_{w, 0}}}^{z 0}$ & ${\overline{\tau_{w}}}^{c} /{\overline{\tau_{w, 0}}}^{z 0}$ \\
\hline $\mathrm{d}: 0$ & 0.75 & 0.96 & 1.00 \\
$\mathrm{p}: 4.5$ & 0.74 & 1.05 & 0.87 \\
$\mathrm{c}: 4.5$ & 0.82 & 0.82 & 0.82 \\
\hline
\end{tabular}

\section{Model of drag reduction in turbulent rectangular ducts}

The results obtained in controlled duct and channel configurations allow to construct an empirical model which provides the expected level of drag reduction for a given set of geometrical parameters, i.e. duct spanwise width $L_{z}$ and width of the controlled region $L_{c}$ for a given width of the ramp $\Delta \lambda$. The extrapolation from our results for $A R=3$ to wider ducts is possible because the peak in the wall-shear stress distribution does not strongly depend on the width of the controlled region $L_{c}$ as the comparison of several channels with different $L_{z}$ and $L_{c}$ shows (not shown). Therefore, we take such a peak as a feature common to all spanwise-limited controlled flows with the following control parameters: $T^{+}=125$ and $A^{+}=4.5$. Apart from the peak, we identify two more distinct regions as shown in Fig. 11. Close to the side walls and outside of the controlled region, the wall-shear stress of p:4.5 follows closely that of the uncontrolled duct case d:0. At the boundary between the controlled and uncontrolled regions the wall-shear stress peaks. We call this the penalty region in the following discussion and consider a width of $1.5 \mathrm{~h}$ for it as can be observed in Fig. 11. In the centre of the duct, p:4.5 shows a wall-shear stress very close to the one of the controlled channel c:4.5. For larger aspect ratios, we expect the wall-shear stress in the centre of the controlled duct to converge towards the value of the controlled channel. This region of the duct will be denoted as controlled region.

We extract the regionally-averaged wall-shear stress for the uncontrolled $\left({\overline{\tau_{w}}}^{0}\right)$, penalty $\left({\overline{\tau_{w}}}^{p n}\right)$ and controlled regions $\left({\overline{\tau_{w}}}^{c}\right)$ from p:4.5 and d:0. Those empirical results are summarized in Table 4. Since we expect the partially-controlled duct to agree with the controlled channel in the centre for larger aspect ratios, we use the wall-shear stress of c:4.5 normalized by the wall-shear stress of the uncontrolled channel for the prediction of drag reduction in wider ducts. Also note that the averaged wall-shear stress in the uncontrolled region from the p:4.5 and d:0 cases almost coincides, thus we are able to use the value from the uncontrolled duct configuration for this region. Using these regionally-averaged wall-shear stresses, we 


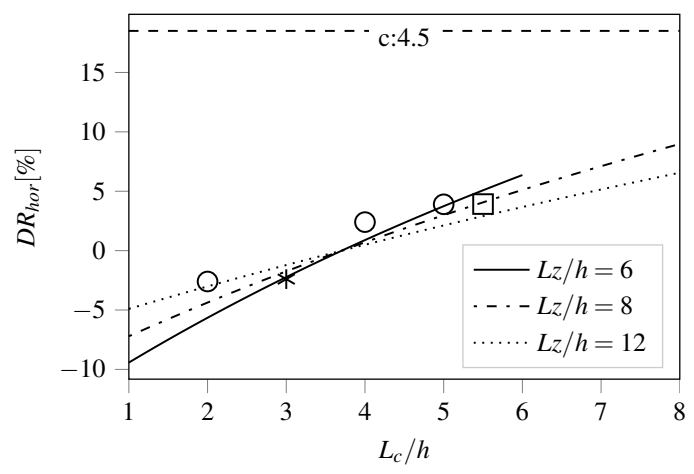

Fig. 12 Prediction of drag reduction along the horizontal walls for ducts and channels of different spanwise width $L_{z} / h$ and controlled width $L_{c} / h$. Markers correspond to $\mathrm{p}: 4.5(*)$, partially-controlled channel simulations at $L_{z} / h=8$ (०) and $L_{z} / h=12(\square)$

construct the model equation

$$
D R_{h o r}\left(L_{0}, L_{c}\right)=1-\frac{0.75 \cdot\left(2 L_{0} / h-1\right)+1.05 \cdot(3)+0.82 \cdot\left(L_{c} / h-2\right)}{0.75 \cdot\left(2 L_{0} / h-1\right)+0.96 \cdot(3)+1.00 \cdot\left(L_{c} / h-2\right)},
$$

where the nominally uncontrolled region $2 L_{0} / h$ is reduced to $2 L_{0} / h-2 \cdot 0.5$ and the controlled region $L_{c} / h$ is reduced to $L_{c} / h-2 \cdot 1$ to account for the width of the penalty region as shown in Fig. 11. This equation can be cast into a more compact form by replacing $L_{0}$ with $\left(L_{z}-L_{c}\right) / 2$ and rearanging terms to

$$
D R_{h o r}\left(L_{z}, L_{c}\right)=1-\frac{0.75 \cdot L_{z} / h+0.08 \cdot L_{c} / h+0.76}{0.75 \cdot L_{z} / h+0.25 \cdot L_{c} / h+0.13}
$$

which allows an a priori prediction of the drag reduction on the horizontal walls for given total width $L_{z}$ and controlled width $L_{c}$. Note that drag reduction or increase at the side walls is not accounted for since for large $A R$ the contribution of the side walls becomes negligible.

The accuracy of this prediction is tested with the four additional partially-controlled channel configurations of $L_{z} / h=8$ and $L_{z} / h=12$ shown in Fig. 12. The model equation accurately predicts the achieved level of drag reduction for $\mathrm{p}: 4.5$ despite using the wall-shear stress of d:0 and c:4.5 in the uncontrolled and controlled regions, respectively. As illustrated in Fig. 12, the model equation roughly predicts the expected level of drag reduction in the additional partially-controlled channel configurations and correctly reflects the general tendency of larger $D R$ values for increasing $L_{z} / h$. Furthermore, we see that the drag reduction obtained in a spanwise-unlimited controlled channel c:4.5 (18.5\%) is more than four times larger than in a partially-controlled channel with $L_{c} / h=5.5$ and $L_{z} / h=12(4 \%)$.

Even though this model equation is limited to $R e_{\tau}=180$ and the present choice of control parameters, it is a useful tool to design flow-control experiments in duct flows, as it provides an estimation of the amount of drag reduction to be expected. In particular, the proposed model can be used as the basis to estimate drag reduction levels obtained in experimental duct facilities at different operating conditions (Reynolds number, aspect ratio, controlled region and control parameters). The following elements would be required for such predictions:

1. A channel flow simulation with the desired control parameters (oscillation amplitude, controlled region, etc.) and Reynolds number, which will provide the wall-shear stress in the controlled region as well as in the penalty region.

2. An uncontrolled duct simulation at the desired Reynolds number, from which the value of the wall-shear stress in the uncontrolled region will be extracted. 


\section{Conclusions}

The influence of spanwise oscillating walls on the overall drag in turbulent duct flows is investigated. It is shown that at the present aspect ratio of $A R=3$ spanwise wall oscillation is hardly successful in reducing skin-friction drag. For low-oscillation amplitudes small overall drag reduction can be realized, whereas drag strongly increases for large-oscillation amplitudes, which are otherwise known to yield large values of drag reduction in turbulent channel flows. The observed drag increase is strongly related to the generation of secondary motions produced at the edges of the oscillating region, where the abrupt change in spanwise wall-velocity generates wall-normal velocity fluctuations owing to continuity. Due to the asymmetric behaviour of the flow at the edges of the controlled region, they do not cancel in a temporal average. Such wall-normal velocity fluctuations cause locally a larger value of wall-shear stress, which is detrimental to the globally achievable skin-friction reduction. As a result, lower drag reduction values compared to periodic channels are achieved, even though the centre of the controlled region exhibits values of drag reduction similar to those achieved in channels.

We distinguish between the case in which the controlled region extends up to the side walls and the case in which only a fraction of the duct width is controlled. In the former, the flow pattern given by the secondary vortices of Prandtl's second kind is strongly modified by the control-induced velocity fluctuations close to the duct corners. In the latter, Prandtl's second kind secondary vortices are very similar to the uncontrolled duct flow, at least in the vicinity of the duct corners. However additional secondary vortices are generated above the spanwise boundaries of the controlled region, as a consequence of the local ejection events. The generated secondary vortices are much stronger than secondary vortices of Prandtl's second kind classically described for duct flows.

Since any technical realisation of an oscillating wall will have to deal with the limited spanwise extent of the oscillating region (unless the oscillations are imposed on a turbulent pipe flow [21]), we propose a simple model that allows an estimation of the drag reduction performance when only a part of the wall is made to oscillate. 


\section{A Validation}

In order to validate the implementation of the oscillating wall, the DNS results of one uncontrolled and one controlled turbulent channel flow simulated in Nek5000 are compared against literature data from Moser, Kim and Mansour (MKM) [39] and results obtained with a classic pseudo-spectral DNS code (abbreviated in the following with PSC) [30]. The profile of the mean streamwise velocity $\bar{u}^{+}$is shown in Fig. 13 (a). Very good agreement between the present results (c:0), data from MKM and results generated with the second code (PSC:0), is found for the uncontrolled case (lower curves). Also the controlled cases show very good agreement. Note that the results are scaled with the actual friction velocity of each flow [2].

(a)
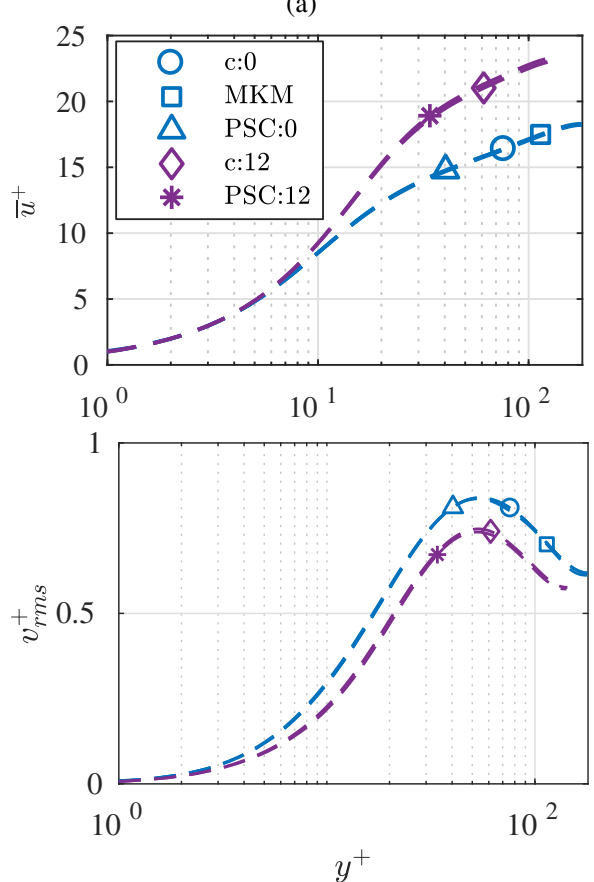

(c) (b)
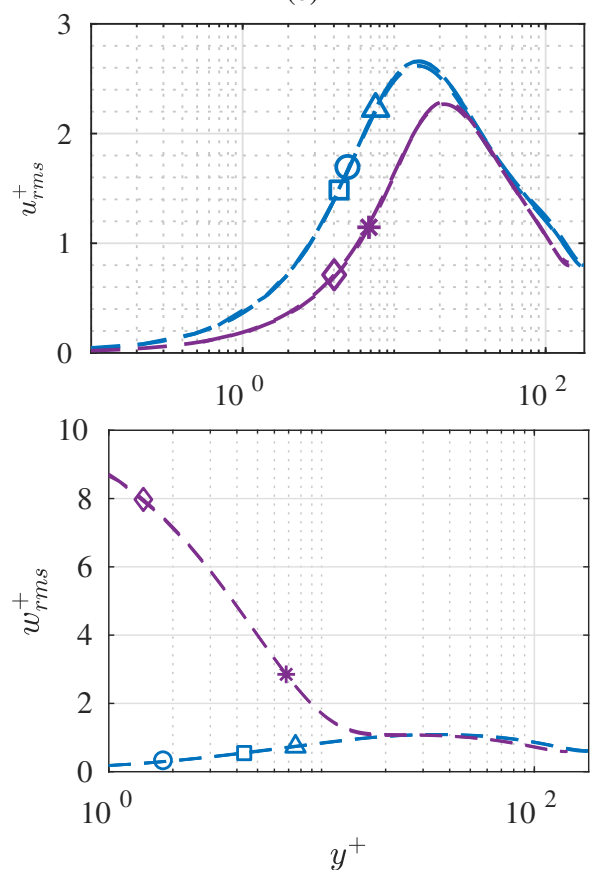

(d)

Fig. 13 Inner-scaled (a) mean streamwise velocity $\bar{u}^{+}$, (b) RMS of streamwise velocity fluctuations, (c) RMS of wall-normal velocity fluctuations and (d) RMS of spanwise velocity fluctuations in the channel compared to literature references [39] (MKM) and results generated with a pseudo-spectral code [30] (PSC). Blue lines correspond to uncontrolled flows and purple lines to controlled flows with $A^{+}=12$

The RMS values of the streamwise velocity fluctuations are presented in Fig. 13 (b). Here, the applied control technique causes a reduction of the inner-scaled streamwise velocity fluctuations close to the wall up to $y^{+} \approx 30$. A slight undershoot of the peak value for $\mathrm{c}: 0$ is visible for both controlled and uncontrolled cases which might be caused by a slightly insufficient spatial resolution [40].

Figure 13 (c) shows the RMS of wall-normal velocity fluctuations. The agreement between uncontrolled channel cases c:0, MKM and CPL:0 is very good, as is the agreement between the two controlled cases c:12 and CPL:12. Excellent agreement is also found for the spanwise velocity fluctuations shown in Fig. 13 (d). The large fluctuations at the wall for the controlled cases are a result of the applied decomposition into mean and fluctuating values (see section 2) since the value at the wall is determined by the prescribed oscillation amplitude. Finally, we compare the Reynolds stresses $-\overline{u^{\prime} v^{\prime}}$ in Figure 14 which shows reasonable agreement for all considered cases. 


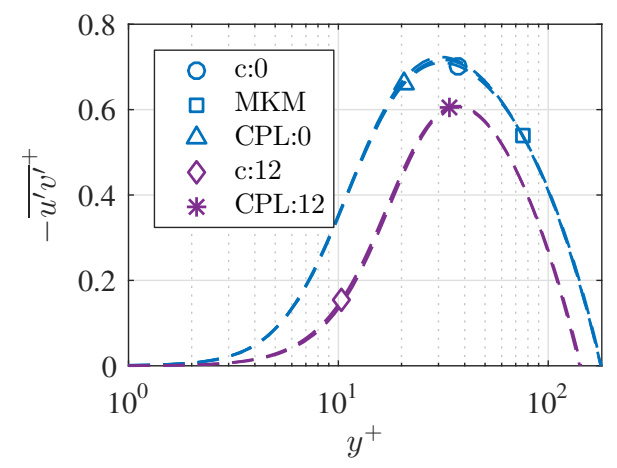

Fig. 14 Inner-scaled Reynolds shear stress in the channel. See Figure 13 for different line styles

Acknowledgements This research was conducted using the resources provided by the Swedish National Infrastructure for Computing (SNIC) at High Performance Computing Center North (HPC2N) and at National Supercomputer Center (NSC) at Linköping University. Steffen Straub is grateful for the financial support of the Prof. Dr.-Ing. Erich Müller-Stiftung and the Dr.-Ing. Willy-Höfler-Stiftung.

Ricardo Vinuesa and Phlipp Schlatter acknowledge the support by the Knut and Alice Wallenberg Foundation and the Swedish Research Council (VR).

Bettina Frohnapfel acknowledges the support by the Alexander von Humboldt Foundation in form of a Feodor-Lynen-Fellowship.

Conflict of Interest: The authors declare that they have no conflict of interest.

\section{References}

1. G. E. Karniadakis and K.-S. Choi. Mechanisms on transverse motion in turbulent wall flows. Annu. Rev. Fluid Mech., 35:45-62, 2003.

2. M. Quadrio. Drag reduction in turbulent boundary layer by in-plane wall motion. Philos. T. R. Soc. A, 369(1940):1428-1442, 2011.

3. M. Quadrio and P. Ricco. Critical assessment of turbulent drag reduction through spanwise wall oscillations. J. Fluid Mech., 521:251-271, 2004.

4. M. Quadrio, P. Ricco, and C. Viotti. Streamwise-traveling waves of spanwise wall velocity for turbulent drag reduction. J. Fluid Mech., 627:161-178, 2009.

5. P. Ricco and S. Hahn. Turbulent drag reduction through rotating discs. J. Fluid Mech., 722:267-290, 5 2013. ISSN 1469-7645.

6. D. J. Wise and P. Ricco. Turbulent drag reduction through oscillating discs. J. Fluid Mech., 746:536-564, 5 2014. ISSN 1469-7645.

7. C.A. Duque-Daza, M.F. Baig, D.A. Lockerby, S.I. Chernyshenko, and C. Davies. Modelling turbulent skin-friction control using linearised Navier-Stokes equations. J. Fluid Mech., 702:403-414, 2012.

8. Davide Gatti and Maurizio Quadrio. Reynolds-number dependence of turbulent skin-friction drag reduction induced by spanwiseforcing. J. Fluid Mech., 802:553-582, 0092016.

9. E. Hurst, Q. Yang, and Y.M. Chung. The effect of Reynolds number on turbulent drag reduction by streamwise travelling waves. J. Fluid Mech., 759:28-55, 11 2014. ISSN 1469-7645.

10. R. Moarref and M. R. Jovanovic. Model-based design of transverse wall oscillations for turbulent drag reduction. J. Fluid Mech., 707:205-240, 2012.

11. L. Agostini, E. Touber, and M.A. Leschziner. The turbulence vorticity as a window to the physics of friction-drag reduction by oscillatory wall motion. Int. J. Heat Fluid Fl., 51:3 - 15, 2015. ISSN 0142727X. Theme special issue celebrating the 75th birthdays of Brian Launder and Kemo Hanjalic.

12. L. Agostini, E. Touber, and M. A. Leschziner. Spanwise oscillatory wall motion in channel flow: dragreduction mechanisms inferred from DNS-predicted phase-wise property variations at $R e_{\tau}=1000 . J$. Fluid Mech., 743:606-635, 0032014.

13. E. Touber and M. A. Leschziner. Near-wall streak modification by spanwise oscillatory wall motion and drag-reduction mechanisms. J. Fluid Mech., 693:150-200, 2012.

14. A. Yakeno, Y. Hasegawa, and N. Kasagi. Modification of quasi-streamwise vortical structure in a dragreduced turbulent channel flow with spanwise wall oscillation. Phys. Fluids, 26(085109):085109, 2014. 
15. Davide Gatti, Andreas Güttler, Bettina Frohnapfel, and Cameron Tropea. Experimental assessment of spanwise-oscillating dielectric electroactive surfaces for turbulent drag reduction in an air channel flow. Exp. Fluids, 56(5):110, 2015.

16. K. Gouder, M. Potter, and J. F. Morrison. Turbulent friction drag reduction using electroactive polymer and electromagnetically driven surfaces. Exp. Fluids, 54(1441):1441, 2013.

17. K.S. Choi. Near-wall structure of turbulent boundary layer with spanwise-wall oscillation. Phys. Fluids, 14(7):2530-2542, 2002.

18. K.-S. Choi, J.-R. DeBisschop, and B. R. Clayton. Turbulent boundary-layer control by means of spanwise-wall oscillation. AIAA Journal, 36(7):1157-1163, 1998.

19. P. Ricco and S. Wu. On the effects of lateral wall oscillations on a turbulent boundary layer. Exp. Therm. Fluid Science, 29:41-52, 2004.

20. S.M. Trujillo, D.G. Bogard, and K.S. Ball. Turbulent Boundary Layer Drag Reduction using an Oscillating Wall. AIAA Paper 97-1870, 1997.

21. F. Auteri, A. Baron, M. Belan, G. Campanardi, and M. Quadrio. Experimental assessment of drag reduction by traveling waves in a turbulent pipe flow. Phys. Fluids, 22(11):115103, 2010.

22. K-S. Choi and M. Graham. Drag reduction of turbulent pipe flows by circular-wall oscillation. Phys. Fluids, 10(1):7-9, 1998.

23. M. Skote. Temporal and spatial transients in turbulent boundary layer flow over an oscillating wall. Int. J. Heat Fluid Fl., 38:1 - 12, 2012. ISSN 0142-727X.

24. S. Lardeau and M. A. Leschziner. The streamwise drag-reduction response of a boundary layer subjected to a sudden imposition of transverse oscillatory wall motion. Phys. Fluids, 25(075109), 2013.

25. M. Skote. Comparison between spatial and temporal wall oscillations in turbulent boundary layer flows. J. Fluid Mech., 730:273-294, 2013.

26. P. F. Fischer, J. W. Lottes, and S. G. Kerkemeier. Nek5000: Open source spectral element CFD solver. Available at http://nek5000.mcs.anl.gov, 2008.

27. A. Patera. A spectral element method for fluid dynamics: Laminar flow in a channel expansion. $J$. Comput. Phys., 54:468-588, 1984.

28. O. Marin, R. Vinuesa, A. V. Obabko, and P. Schlatter. Characterization of the secondary flow in hexagonal ducts. Phys. Fluids, 28(12):125101, 2016.

29. J. Kim, P. Moin, and R. Moser. Turbulence statistics in fully developed channel flow at low Reynolds number. J. Fluid Mech., 177:133-166, 1987.

30. P. Luchini and M. Quadrio. A low-cost parallel implementation of direct numerical simulation of wall turbulence. J. Comput. Phys., 211:551-571, 2006.

31. R. Vinuesa, A. Noorani, A. Lozano-Durn, G. K. El Khoury, P. Schlatter, P. F. Fischer, and H. M. Nagib. Aspect ratio effects in turbulent duct flows studied through direct numerical simulation. J. Turbul., 15 (10):677-706, 2014.

32. Steffen Straub, Ricardo Vinuesa, Philipp Schlatter, Bettina Frohnapfel, and Davide Gatti. Direct numerical simulation of controlled turbulent duct flows. Master's thesis, Karlsruhe Institute of Technology, 2015.

33. P. Ricco and M. Quadrio. Wall-oscillation conditions for drag reduction in turbulent channel flow. Int. J. Heat Fluid Fl., 29:601-612, 2008.

34. Ricardo Vinuesa, Cezary Prus, Philipp Schlatter, and Hassan M. Nagib. Convergence of numerical simulations of turbulent wall-bounded flows and mean cross-flow structure of rectangular ducts. Meccanica, 51(12):3025-3042, 2016. ISSN 1572-9648.

35. Adrián Lozano-Durán and Javier Jiménez. Effect of the computational domain on direct simulations of turbulent channels up to re =4200. Physics of Fluids, 26(1):011702, 2014.

36. R. Vinuesa, P. Schlatter, and H. M. Nagib. Characterization of the secondary flow in turbulent rectangular ducts with varying aspect ratio. In Int. Symp. Turbulence \& Shear Flow Phenomena (TSFP-9), 30 June - 3 July, Melbourne, Australia., 2015.

37. K. Fukagata, K. Iwamoto, and N. Kasagi. Contribution of Reynolds stress distribution to the skin friction in wall-bounded flows. Phys. Fluids, 14(11):L73-76, 2002.

38. Todd A. Oliver, Nicholas Malaya, Rhys Ulerich, and Robert D. Moser. Estimating uncertainties in statistics computed from direct numerical simulation. Phys. Fluids, 26(3):035101, 2014.

39. R. D. Moser, J. Kim, and N. N. Mansour. Direct numerical simulation of turbulent channel flow up to $R e_{\tau}=$ 590. Phys. Fluids, 11(4):943-945, 1999

40. R. Örlü and P. Schlatter. Comparison of experiments and simulations for zero pressure gradient turbulent boundary layers at moderate Reynolds numbers. Exp. Fluids, 54(6):1547, 2013. 